PROCEEDINGS OF THE

AMERICAN MATHEMATICAL SOCIETY

Volume 138, Number 7, July 2010, Pages 2561-2567

S 0002-9939(10)10316-5

Article electronically published on February 25, 2010

\title{
ON THE GAUSS CURVATURE OF COMPACT SURFACES IN HOMOGENEOUS 3-MANIFOLDS
}

\author{
FRANCISCO TORRALBO AND FRANCISCO URBANO
}

(Communicated by Jon G. Wolfson)

\begin{abstract}
Compact flat surfaces of homogeneous Riemannian 3-manifolds with isometry group of dimension 4 are classified. Nonexistence results for compact constant Gauss curvature surfaces in these 3-manifolds are established.
\end{abstract}

\section{INTRODUCTION}

The study of complete constant Gauss curvature surfaces in simply connected 3manifolds of constant curvature is a classical topic in classical differential geometry. Perhaps one of the nicest reviews about it can be found in Spivak's book 7 .

During the last lustrum, the surfaces of homogeneous Riemannian 3-manifolds were being deeply studied and particularly those with constant mean curvature. The starting point of this study was the work of Abresch and Rosenberg [1, where they found a holomorphic quadratic differential in any constant mean curvature surface of a homogeneous Riemannian 3-manifold with isometry group of dimension 4. The Berger 3 -spheres, the Heisenberg group, the Lie group $S l(2, \mathbb{R})$ and the Riemannian products $\mathbb{S}^{2} \times \mathbb{R}$ or $\mathbb{H}^{2} \times \mathbb{R}$, where $\mathbb{S}^{2}$ and $\mathbb{H}^{2}$ are the 2-dimensional sphere and hyperbolic plane with their standard metrics, are the most significant examples of such homogeneous 3-manifolds.

In important and complete works [2, 3, Aledo, Espinar and Gálvez classified the complete constant Gauss curvature surfaces in $\mathbb{S}^{2} \times \mathbb{R}$ and $\mathbb{H}^{2} \times \mathbb{R}$ for any value of the Gauss curvature except for several values, where the classification is still open.

In this paper we start the study of the Gauss curvature of compact surfaces of homogeneous Riemannian 3-manifolds with isometry group of dimension 4 . We get a nice integral formula (formula (3.3)), which allows us to classify the flat compact surfaces (Theorem 3.1). This formula is new in the sense that there is not a similar one for surfaces in 3-dimensional space forms, and it shows an important difference between flat compact surfaces in these 3-manifolds and in 3-dimensional space forms. For instance, in the Berger spheres the only flat compact surfaces are the Hopf tori, i.e., inverse image of closed curves of $\mathbb{S}^{2}$ by the Hopf fibration $\Pi: \mathbb{S}^{3} \rightarrow \mathbb{S}^{2}$, whereas in the standard 3 -sphere, besides them, there are other flat tori (see [8]).

Received by the editors March 12, 2009, and, in revised form, October 20, 2009.

2010 Mathematics Subject Classification. Primary 53C42; Secondary 53C30.

This research was partially supported by MCyT-Feder research project MTM2007-61775 and Junta Andalucía Grant P06-FQM-01642.

(c) 2010 American Mathematical Society 
Using arguments of a topological nature, in Theorem 3.6 and Corollary 3.7, we study the behavior of the Gauss curvature of this class of surfaces and we prove nonexistence results of compact surfaces with constant Gauss curvature in these homogeneous Riemannian 3-manifolds.

\section{Preliminaries}

In this section we follow the notation given in [4. Let $N$ be a simply connected homogeneous Riemannian 3-manifold whose isometry group has dimension 4 . Then there exists a Riemannian submersion $\Pi: N \rightarrow M^{2}(\kappa)$, where $M^{2}(\kappa)$ is a 2dimensional simply connected space form of constant curvature $\kappa$, with totally geodesic fibers and there exists a unit Killing field $\xi$ on $N$ which is vertical with respect to $\Pi$. We will assume that $N$ is oriented, and then we can define a vectorial product $\wedge$, such that if $\left\{e_{1}, e_{2}\right\}$ are linearly independent vectors at a point $p$, then $\left\{e_{1}, e_{2}, e_{1} \wedge e_{2}\right\}$ is the orientation at $p$. If $\bar{\nabla}$ denotes the Riemannian connection on $N$, the properties of $\xi$ imply (see [4) that for any vector field $V$,

$$
\bar{\nabla}_{V} \xi=\tau(V \wedge \xi)
$$

where the constant $\tau$ is the bundle curvature. As the isometry group of $N$ has dimension $4, \kappa-4 \tau^{2} \neq 0$. The case $\kappa-4 \tau^{2}=0$ corresponds to $\mathbb{S}^{3}$ with its standard metric if $\tau \neq 0$ and to the Euclidean space $\mathbb{R}^{3}$ if $\tau=0$, which have isometry groups of dimension 6 .

When $\tau=0, N$ is the product $M^{2}(\kappa) \times \mathbb{R}$. Its quotient $M^{2}(\kappa) \times \mathbb{S}^{1}$, where $\mathbb{S}^{1}$ is a circle of a certain radius, also satisfies the same geometrical properties.

When $\tau \neq 0, N$ is one of the Berger spheres for $\kappa>0$, the Heisenberg group $\mathrm{Nil}_{3}$ for $\kappa=0$ and the universal cover $\widehat{\operatorname{Sl}(2, \mathbb{R})}$ of the Lie group $\operatorname{Sl}(2, \mathbb{R})$ for $\kappa<0$. The parameters $\kappa, \tau$ give a 2-parameter family of Berger metrics on $\mathbb{S}^{3}$ and of homogeneous metrics on $\widehat{S l(2, \mathbb{R})}$. The parameter $\tau$ gives a 1-parameter family of homogeneous metrics on $\mathrm{Nil}_{3}$.

The real projective space $\mathbb{R} \mathbb{P}^{3}$ or more generally the Lens spaces $L_{n}=\mathbb{S}^{3} / \mathbb{Z}_{n}, n \geq$ 3 , with the induced Berger metrics are also homogeneous Riemannian 3-manifolds with isometry group of dimension 4 . Also, $\operatorname{PSl}(2, \mathbb{R})=S l(2, \mathbb{R}) /\{ \pm I\}$ or more generally $S l(2, \mathbb{R}) / \mathbb{Z}_{n}, n \geq 3$, are homogeneous Riemannian 3-manifolds with isometry group of dimension 4 too. In these cases, the corresponding Riemannian submersion on $M^{2}(\kappa)$ is by circles.

Throughout the paper, $E(\kappa, \tau)$ will denote either a simply connected homogeneous Riemannian 3-manifold with isometry group of dimension 4 or some of the above quotients, where $\kappa$ is the curvature of the basis, $\tau$ the bundle curvature (and therefore $\kappa-4 \tau^{2} \neq 0$ ).

Now, let $\Phi: \Sigma \rightarrow E(\kappa, \tau)$ be an immersion of an orientable surface $\Sigma$ and $N$ a unit normal field on $\Sigma$. We define a function $C$ on $\Sigma$ by

$$
C=\langle\xi, N\rangle
$$

where $\langle$,$\rangle stands for the metric on both E(\kappa, \tau)$ and $\Sigma$. It is clear that $-1 \leq C \leq 1$ and that, when $\tau \neq 0,\left\{p \in \Sigma \mid C^{2}(p)=1\right\}=\{p \in \Sigma \mid \xi(p)= \pm N(p)\}$ has empty interior, because the distribution $\langle\xi\rangle^{\perp}$ on $E(\kappa, \tau)$ is not integrable. If $A$ is the shape operator of $\Phi$ associated to $N$ and $K$ is the Gauss curvature of $\Sigma$, then the Gauss 
equation of $\Phi$ is given by (see [4)

$$
K=\operatorname{det} A+\tau^{2}+\left(\kappa-4 \tau^{2}\right) C^{2}=2 H^{2}-\frac{|\sigma|^{2}}{2}+\tau^{2}+\left(\kappa-4 \tau^{2}\right) C^{2},
$$

where $H$ is the mean curvature associated to $N$ and $\sigma$ is the second fundamental form of $\Phi$.

As there exists a Riemannian submersion $\Pi: E(\kappa, \tau) \rightarrow M^{2}(\kappa)$, we can construct flat surfaces in $E(\kappa, \tau)$ in the following way. Given any regular curve $\gamma$ in $M^{2}(\kappa)$, $\Pi^{-1}(\gamma)$ is a surface in $E(\kappa, \tau)$ which has $\xi$ as a tangent vector field, i.e., $C=0$. From (2.1) it follows that $\xi$ is a parallel vector field on $\Sigma$ and hence $\Pi^{-1}(\gamma)$ is a flat surface. We will call $\Pi^{-1}(\gamma)$ a Hopf surface of $E(\kappa, \tau)$. If $\gamma$ is a closed curve, then $\Pi^{-1}(\gamma)$ is a flat cylinder; and moreover if $\Pi$ is a circle Riemannian submersion, then $\Pi^{-1}(\gamma)$ is a flat torus.

\section{Statement of Results}

We start by classifying the compact flat surfaces of $E(\kappa, \tau)$.

Theorem 3.1. The only flat compact surfaces in $E(\kappa, \tau)$ are the Hopf tori. In particular,

- In $M^{2}(\kappa) \times \mathbb{R}, \kappa \neq 0$, the Heisenberg group $\mathrm{Nil}_{3}$ and $\widetilde{S l(2, \mathbb{R})}$, there are no flat compact surfaces.

- In $M^{2}(\kappa) \times \mathbb{S}^{1}, \kappa \neq 0$, the only flat compact surfaces are the products $\gamma \times \mathbb{S}^{1}$, where $\gamma$ is a closed curve in $M^{2}(\kappa)$.

- In the Berger spheres, $S l(2, \mathbb{R})$ and their quotients $L_{n}$ and $S l(2, \mathbb{R}) / \mathbb{Z}_{n}$, the flat compact surfaces are the Hopf tori.

Remark 3.2. The result in the Berger spheres contrasts with the case of the round sphere, where, besides the Hopf tori, there are other flat tori (see [8]).

Proof. First we are going to prove an integral formula for any compact surface of $E(\kappa, \tau)$. Let $\Phi: \Sigma \rightarrow E(\kappa, \tau)$ be an immersion of an oriented compact surface $\Sigma$ and $X$ the tangential component of the Killing field $\xi$, i.e.

$$
X=\xi-C N \text {. }
$$

Then from (2.1) and for any tangent vector $v$ to $\Sigma$ we obtain that

$$
\nabla_{v} X=\tau C(v \wedge N)+C A v
$$

where $\nabla$ is the Riemannian connection on $\Sigma$. From (3.1) we obtain that

$$
\operatorname{div} X=2 C H, \quad d\left(X^{\beta}\right)(v, w)=2 \tau C\langle v \wedge N, w\rangle,
$$

where div is the divergence operator and $X^{\beta}$ is the 1-form associated to $X$, i.e., $X^{\beta}(v)=\langle X, v\rangle$. Now the classical Bochner formula says that

$$
\operatorname{div}\left(\nabla_{X} X\right)=K|X|^{2}+\langle\nabla(\operatorname{div} X), X\rangle+|\nabla X|^{2}+\sum_{i=1}^{2} d\left(X^{\beta}\right)\left(\nabla_{e_{i}} X, e_{i}\right),
$$

where $\left\{e_{1}, e_{2}\right\}$ is an orthonormal basis in $T \Sigma$. Using (3.1) and (3.2), this equation becomes

$$
\operatorname{div}\left(\nabla_{X} X\right)=K\left(1-C^{2}\right)+\langle\nabla(\operatorname{div} X), X\rangle+C^{2}|\sigma|^{2}-2 \tau^{2} C^{2} .
$$

From (3.2) we get that

$$
\operatorname{div}(2 C H X)=\operatorname{div}((\operatorname{div} X) X)=\langle\nabla(\operatorname{div} X), X\rangle+(\operatorname{div} X)^{2} .
$$


The last two formulae joined with (3.2) again give

$$
\operatorname{div}\left(\nabla_{X} X-2 C H X\right)=K\left(1-C^{2}\right)-4 H^{2} C^{2}+C^{2}|\sigma|^{2}-2 \tau^{2} C^{2} .
$$

Using the Gauss equation (2.2) in the above formula, we obtain

$$
\operatorname{div}\left(\nabla_{X} X-2 C H X\right)=K\left(1-3 C^{2}\right)+2\left(\kappa-4 \tau^{2}\right) C^{4} .
$$

Finally, using the divergence theorem, the last formula becomes the following integral formula for any orientable compact surface of $E(\kappa, \tau)$ :

$$
\int_{\Sigma} K\left(1-3 C^{2}\right) d A+2\left(\kappa-4 \tau^{2}\right) \int_{\Sigma} C^{4} d A=0 .
$$

Although the function $C$ is only well defined for orientable surfaces, it is clear that $C^{2}$ is well defined always, even for nonorientable surfaces. So formula (3.3) is true for any compact surface of $E(\kappa, \tau)$.

Now we prove the theorem. If $K=0$, (3.3) becomes

$$
\left(\kappa-4 \tau^{2}\right) \int_{\Sigma} C^{4} d A=0,
$$

which implies that $C=0$ on $\Sigma$. This means that $X=\xi$, and so the vertical Killing field of the fibration $\Pi: E(\kappa, \tau) \rightarrow M^{2}(\kappa)$ is tangent to $\Sigma$. So $\Pi(\Sigma)$ is a closed curve $\gamma$ in $M^{2}(\kappa)$ and then $\Pi^{-1}(\gamma) \supset \Sigma$, which proves that $\Sigma$ is the Hopf torus $\Pi^{-1}(\gamma)$.

Remark 3.3. Formula (3.3) is also true for compact surfaces in other 3-manifolds. In fact, suppose that $\Sigma$ is a compact surface of the Riemannian product $M^{2} \times \mathbb{R}$ or $M^{2} \times \mathbb{S}^{1}$, where $M^{2}$ is an oriented surface. In this case, $\Pi: M^{2} \times \mathbb{R} \rightarrow M^{2}$ is the trivial submersion and so $\xi=(0,1)$ is a parallel vector field. Following the proof of (3.3), if $X$ is the tangential component of $\xi$, then $\operatorname{div} X=2 C H, d X^{\beta}=0$ and $\operatorname{div}\left(\nabla_{X} X-2 C H X\right)=K\left(1-3 C^{2}\right)+\bar{K} C^{4}$, where $\bar{K}$ is the Gauss curvature of the surface $M^{2}$. Now the divergence theorem says that

$$
\int_{\Sigma}\left(K\left(1-3 C^{2}\right)+2 \bar{K} C^{4}\right) d A=0
$$

Hence following the proof of Theorem 3.1 we can prove:

Let $M^{2}$ be an oriented surface with Gauss curvature $\bar{K}>0$ or $\bar{K}<0$. Then:

(1) There exist no compact flat surfaces in $M^{2} \times \mathbb{R}$.

(2) The only compact flat surfaces in $M^{2} \times \mathbb{S}^{1}$ are the products $\gamma \times \mathbb{S}^{1}$, where $\gamma$ is a closed curve in $M^{2}$.

Corollary 3.4. The only compact surfaces in $E(\kappa, \tau)$ with $C$ constant and $C^{2} \neq 1$ are the Hopf tori, for which $C=0$.

\section{Remark 3.5.}

(1) This result is not true for noncompact surfaces. In fact, in [5, Leite constructed for each $\left.K_{0} \in\right]-1,0[$ a constant mean curvature embedding of the hyperbolic plane with its standard metric of negative constant curvature $K_{0}$ in $M^{2}(-1) \times \mathbb{R}$, with constant function $C=-K_{0}$.

(2) When $C^{2}=1$ it is well known, even without the compactness assumption, that $\tau=0$ and the surface is a totally geodesic slice of $M^{2}(\kappa) \times \mathbb{R}$. 
Proof. As $C^{2} \neq 1$, then the tangential component $X$ of the Killing field $\xi$ is a vector field on $\Sigma$ without zeros. So the Euler-Poincaré characteristic $\chi(\Sigma)=0$ and then $\int_{\Sigma} K d A=0$. Now, as $C$ is constant, formula (3.3) becomes $\left(\kappa-4 \tau^{2}\right) C^{4}$ Area $(\Sigma)=$ 0 , and hence $C=0$. We finish as in the proof of Theorem 3.1 .

Theorem 3.6. a) There exist no compact surfaces in $E(\kappa, \tau)$ with Gauss curvature $K<\min \left\{0, \kappa-4 \tau^{2}\right\}$.

b) Let $\Phi: \Sigma \rightarrow E(\kappa, \tau)$ be an immersion of a compact surface $\Sigma$ with Gauss curvature $K$.

(1) If $\kappa-4 \tau^{2}>0$ and $0 \leq K<\max \left\{\kappa-4 \tau^{2}, \tau^{2}\right\}$, then $K=0$ and $\Sigma$ is a Hopf torus.

(2) If $\kappa-4 \tau^{2}<K \leq 0$, then $K=0$ and $\Sigma$ is a Hopf torus.

(3) If $\kappa-4 \tau^{2}<0 \leq K<\kappa-3 \tau^{2}$ ( $\kappa$ must be positive), then $K=0$ and $\Sigma$ is a Hopf torus.

Proof. First we prove a). Considering the double oriented cover, if necessary, we can assume that the surface $\Sigma$ is orientable. Suppose that $\Phi: \Sigma \rightarrow E(\kappa, \tau)$ is an immersion of an orientable compact surface with Gauss curvature $K<\min \{0, \kappa-$ $\left.4 \tau^{2}\right\}$. Let $X$ be the tangential component of the Killing field $\xi$. From (2.1) and for any tangent vector $v$ to $\Sigma$, we get

$$
\tau(v \wedge X)=\sigma(v, X)+\langle\nabla C, v\rangle N,
$$

and so

$$
\nabla C=\tau(X \wedge N)-A X .
$$

It is clear that the points $p$ with $C^{2}(p)=1$, that is, $X_{p}=0$, are critical points of $C$. Suppose that $p$ is a critical point of $C$ with $C^{2}(p)<1$. As $X_{p} \neq 0$, equation (3.4) says that $A X_{p}=\tau\left(X_{p} \wedge N_{p}\right)$ and hence det $A(p)=-\tau^{2}$. The Gauss equation (2.2) implies that $K(p)=\left(\kappa-4 \tau^{2}\right) C^{2}(p)$. As $0 \leq C^{2}(p) \leq 1$, the assumptions on the Gauss curvature mean that such a critical point $p$ of $C$ cannot exist. Hence the only critical points of $C$ are those with $C^{2}(p)=1$.

From (3.4) and (3.1) it is not difficult to check that the Hessian of $C$ at a critical point $p$, where $C^{2}(p)=1$ and $X_{p}=0$, is given by

$$
\begin{array}{r}
\left(\nabla^{2} C\right)(v, w)=-C(p)\left\langle A^{2} v, w\right\rangle-\tau^{2} C(p)\left\langle v \wedge N_{p}, w \wedge N_{p}\right\rangle \\
-\tau C(p)\left\langle A v \wedge w+A w \wedge v, N_{p}\right\rangle,
\end{array}
$$

where $v, w$ are tangent vectors to $\Sigma$ at $p$. Hence, if $\left\{e_{1}, e_{2}\right\}$ is an orthonormal basis of $T_{p} \Sigma$ with $A e_{i}=\lambda_{i} e_{i}, i=1,2$, the determinant of the Hessian of $C$ at $p$ is given by

$$
\operatorname{det}\left(\nabla^{2} C\right)(p)=\left(\lambda_{1} \lambda_{2}+\tau^{2}\right)^{2}=\left(K(p)-\left(\kappa-4 \tau^{2}\right)\right)^{2}>0 .
$$

This means that $C$ is a Morse function on $\Sigma$ with only the absolute maximum and minimum as critical points. Elementary Morse theory says that $\Sigma$ must be a sphere. As $K$ is negative, this contradicts the Gauss-Bonnet theorem. This proves a).

Proof of b). As in case a), we can assume that our surface is orientable.

First we suppose that either $\kappa-4 \tau^{2}<K \leq 0$ or $0 \leq K<\kappa-4 \tau^{2}$.

Now let $p$ be a zero of the vector field $X$ defined by $X=\xi-C N$. Then following [6], Chapter 6 , if $(\nabla X)_{p}: T_{p} \Sigma \rightarrow T_{p} \Sigma$ denotes the derivative of $X$ at the point $p$, then $p$ is a nondegenerate zero of $X$ if $\operatorname{det}(\nabla X)_{p} \neq 0$. 
From (3.1), if $\left\{e_{1}, e_{2}\right\}$ is an orthonormal basis of $T_{p} \Sigma$, then

$$
\left\langle\nabla_{e_{i}} X, e_{j}\right\rangle=C(p)\left\langle A e_{i}, e_{j}\right\rangle-\tau C(p)\left\langle e_{i} \wedge e_{j}, N(p)\right\rangle,
$$

and so taking into account the Gauss equation (2.2), $\operatorname{det}(\nabla X)_{p}=\operatorname{det} A(p)+\tau^{2}=$ $K(p)-\left(\kappa-4 \tau^{2}\right)$. Using the assumptions on the curvature $K$, this proves that such a zero $p$ of $X$ is nondegenerate. So $X$ has a finite number of zeros.

Now as the index of $X$ at a nondegenerate zero $p$ is 1 (respectively -1 ) if $\operatorname{det}(\nabla X)_{p}>0$ (respectively $\left.\operatorname{det}(\nabla X)_{p}<0\right)$, we have that if $K>\kappa-4 \tau^{2}$ all the zeros of $X$ (if they exist) have index 1, and then the Poincaré-Hopf theorem says that the Euler-Poincaré characteristic $\chi(\Sigma)$ is 2 if $X$ has zeros or $\chi(\Sigma)=0$ if $X$ has no zeros. As $K \leq 0$, the Gauss-Bonnet theorem says that $K=0$ and so Theorem 3.1 proves that $\Sigma$ is a Hopf torus. This proves (2).

If $K<\kappa-4 \tau^{2}$, then all zeros of $X$ (if they exist) have index -1 , and then the Poincaré-Hopf theorem says that $\chi(\Sigma)$ is negative if $X$ has zeros or $\chi(\Sigma)=0$ if $X$ has no zeros. As $K \geq 0$, the Gauss-Bonnet theorem says again that $K=0$ and so $\Sigma$ is a Hopf torus.

Suppose now that $\kappa-4 \tau^{2}>0$ and $0 \leq K<\tau^{2}$.

The Gauss equation of $\Phi$ says that

$$
\tau^{2}>K=\operatorname{det} A+\tau^{2}+\left(\kappa-4 \tau^{2}\right) C^{2} \geq \operatorname{det} A+\tau^{2} .
$$

Hence det $A<0$ on $\Sigma$, and so if $\lambda_{1} \geq \lambda_{2}$ are the principal curvatures of $\Sigma$ associated to $N$, then $\lambda_{2}<0<\lambda_{1}$; and hence the tangent bundle $T \Sigma$ is the direct sum of two rank 1 vector sub-bundles over $\Sigma$, and $T \Sigma=D_{1} \oplus D_{2}$ is defined by $D_{i}(p)=\left\{v \in T_{p} \Sigma \mid A v=\lambda_{i}(p) v\right\}, i=1,2$. As a sphere admits neither vector fields without zeros nor 2-fold covers, such a decomposition is not possible if $\Sigma$ is a sphere. Hence, $\chi(\Sigma) \leq 0$. As $K \geq 0$, the Gauss-Bonnet theorem says that $\Sigma$ is flat, and Theorem 3.1 says that $\Sigma$ is a Hopf torus. This finishes the proof of (1).

Finally suppose that $\kappa-4 \tau^{2}<0 \leq K<\kappa-3 \tau^{2}$. In this case, as $C^{2} \leq 1$, we have

$$
\kappa-3 \tau^{2}>K=\operatorname{det} A+\tau^{2}+\left(\kappa-4 \tau^{2}\right) C^{2} \geq \operatorname{det} A+\kappa-3 \tau^{2} .
$$

Hence $\operatorname{det} A<0$ on $\Sigma$ and the above reasoning proves again that $K=0$ and $\Sigma$ is a Hopf torus. This proves (3).

Corollary 3.7. Let $\Phi: \Sigma \rightarrow E(\kappa, \tau)$ be an immersion of a compact surface $\Sigma$ with constant Gauss curvature $K$.

(1) If $\kappa-4 \tau^{2}>0$ and $\left.K \in\right]-\infty, \max \left\{\kappa-4 \tau^{2}, \tau^{2}\right\}[$, then $K=0$ and $\Sigma$ is a Hopf torus.

(2) If $\kappa-4 \tau^{2}<0<\kappa-3 \tau^{2}$ and $\left.K \in\right]-\infty, \kappa-3 \tau^{2}[$, then either $K=0$ and $\Sigma$ is a Hopf torus or $K=\kappa-4 \tau^{2}$.

(3) If $\kappa-4 \tau^{2}<0$ and $\left.\left.K \in\right]-\infty, 0\right]$, then either $K=0$ and $\Sigma$ is a Hopf torus or $K=\kappa-4 \tau^{2}$.

Remark 3.8. When $\tau=0$, this corollary was proved in 2, 3]. In fact the authors classified the complete constant Gauss curvature surfaces of $M^{2}(\kappa) \times \mathbb{R}, \kappa \neq 0$, except for $K=\kappa$. 


\section{REFERENCES}

1. U. Abresch and H. Rosenberg. A Hopf differential for constant mean curvature surfaces in $\mathbb{S}^{2} \times \mathbb{R}$ and $\mathbb{H}^{2} \times \mathbb{R}$. Acta Math. 193 (2004), 141-174. MR2134864 (2006h:53003)

2. J.A. Aledo, J.M. Espinar and J.A. Gálvez. Complete surfaces of constant curvature in $\mathbb{H}^{2} \times \mathbb{R}$ and $\mathbb{S}^{2} \times \mathbb{R}$. Calculus of Variations and PDE's 29 (2007) 347-363. MR2321892(2008f:53075)

3. J.A. Aledo, J.M. Espinar and J.A. Gálvez. Surfaces with constant curvature in $\mathbb{S}^{2} \times \mathbb{R}$ and $\mathbb{H}^{2} \times \mathbb{R}$. Height estimates and representation. Bull. Braz. Math. Soc. (N.S.) 38 (2007) 533-554. MR2371944 (2008k:53121)

4. B. Daniel. Isometric immersions into 3-dimensional homogeneous manifolds. Comment. Math. Helv. 82 (2007) 87-131. MR 2296059 (2008a:53058)

5. M.L. Leite. An elementary proof of the Abresch-Rosenberg theorem on constant mean curvature immersed surfaces in $\mathbb{S}^{2} \times \mathbb{R}$ and $\mathbb{H}^{2} \times \mathbb{R}$. Quart. J. Math. 58 (2007) 479-487. MR 2371467 (2008j:53013)

6. J.W. Milnor. Topology from the differentiable viewpoint. The University Press of Virginia (1976). MR.1487640 (98h:57051)

7. M. Spivak. A comprehensive introduction to Differential Geometry. Publish or Perish (1979).

8. J.L. Weiner. Flat tori in $\mathbb{S}^{3}$ and their Gauss maps. Proc. London Math. Soc. (3) 62 (1991) 54-76. MR.1078213 (92d:53057)

Departamento de Geometría y Topología, Universidad de Granada, 18071 Granada, SPAIN

E-mail address: ftorralbo@ugr.es

Departamento de Geometría y Topología, Universidad de Granada, 18071 Granada, SPAIN

E-mail address: furbano@ugr.es 